\title{
Semi-Analytical Modelling of Linear Mode Coupling in Few-Mode Fibers
}

\author{
Filipe M. Ferreira, Member, IEEE, Christian S. Costa, Stylianos Sygletos, \\ Andrew D. Ellis, Member, IEEE
}

\begin{abstract}
This paper reviews and extends a method for the semi-analytical solution of the coupled linear differential equations that describe the linear mode coupling arising in fewmode fibers due to waveguide imperfections. The semi-analytical solutions obtained proved to be accurate when compared to numerical solution methods. These solutions were integrated into a multi-section model with split-steps for mode dispersion and mode coupling. Simulations using this model matched the analytical predictions for the statistics of group-delays in fewmode fiber links, considering different coupling regimes with and without mode delay management.
\end{abstract}

Index Terms-Mode-division multiplexing, multimode fiber, few-mode fiber, linear mode coupling.

\section{INTRODUCTION}

$\mathrm{M}$ ODE-DIVISION MULTIPLEXING over few-mode fibers (FMFs) has been proposed as a next-generation solution to overcome the impeding installed capacity exhaustion of current single-mode fibers (SMFs) [1]-[2] However, these systems require significantly higher equalizer complexity given the overall group-delay (GD) spread due to differential mode delay (DMD) and linear mode coupling [2][4]. To correctly estimate the GD spread and the performance of a MDM equalizer, the mode coupling arising from the waveguide imperfections [5], need to be correctly modelled. Thereby, intense research has been accomplished to study the statistics of GDs analytically [6]-[10] and numerically [13][17].

A significant number of works assume systems operating in the strong mode coupling regime, e.g. [6] and [10], and consider a multi-section model where mode coupling is introduced through random unitary matrices each section, where the length of each section must be longer than the correlation length. However, few-mode fibers ([18]-[21])

Manuscript received day month, year; revised day month, year; accepted day month, year. Date of publication day month, year; date of current version day month, year. This work has been partially supported by the European Union (Grants 619732-INSPACE, 654809-HSPACE, and 659950INVENTION), and by the EPSRC (Grant EP/L000091/1-PEACE).

The authors are with the Aston Institute of Photonic Technologies, School of Engineering and Applied Science, Birmingham B4 7ET, U.K. (e-mail: f.ferreira@ason.ac.uk; c.sanchez-costa@aston.ac.uk; s.sygletos@aston.ac.uk; andrew.ellis@aston.ac.uk).

Color versions of one or more of the figures in this paper are available online at http://ieeexplore.iee.org.

Digital Object Identifier X usually operate in the weak or intermediate coupling regime for transmission distances $100-1000 \mathrm{~km}$. Even tough strong mode coupling can be assumed within groups of degenerate modes [11], the mode coupling between groups of nondegenerate modes cannot either be considered negligible or strong. Note that, nonlinear simulation requires a step-size much smaller than nonlinear effective length $(\sim 20 \mathrm{~km})$ [22], thus the generation of coupling matrices with the appropriate level for $10-100 \mathrm{~m}$ is required. Therefore, models considering random unitary matrices do not cover many of the cases of interest.

To model systems operating in the weak and intermediate coupling regime, the introduction of coupling in the form of misaligned fiber splices in each section of a multi-section model was proposed [14]. In this case, the mode coupling matrices are obtained using an overlap integral approach. However, the matrix elements obtained this way present two limitations. First, even though the coefficients are effective in describing the mode power distribution, they fail to consider phase effects thereby appropriate only for incoherent sources [24]. Second, the coupling elements inevitably include mode dependent loss given the nature of the overlap integral. Even though splices do introduce mode dependent loss, splices are here being used as a discrete representation of continuous imperfections which may introduce or not introduce mode dependent loss. Therefore, a model able to separate mode coupling from mode dependent loss is preferable.

Recently, the authors have presented a semi-analytical model capable of describing the linear mode coupling for fibers operating in the intermediate coupling regime [15][16]. Using such method, the authors matched the analytical predictions for group-delay in few-mode fiber links [25]-[26].

In this paper, we review and extend the derivation of a semi-analytical solution method for the linear mode coupling equations, and validate the group-delay spreading predictions for different coupling regimes and different link configurations. Section II presents the coupled differential equations that describe the linear mode coupling and the wellknown solutions for two modes propagation case. Section III presents the semi-analytical solution method proposed for the higher-order modes case including the explicit solutions for three modes and the symbolic computation code for higherorder modes cases. Section IV provides the validation of the semi-analytical solutions obtained for one coupling segment. Section $\mathrm{V}$ described the proposed multi-section model using 
(a)

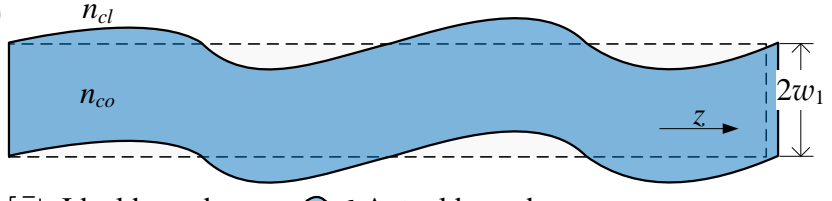

[-I Ideal boundary $\approx$ Actual boundary

(b)

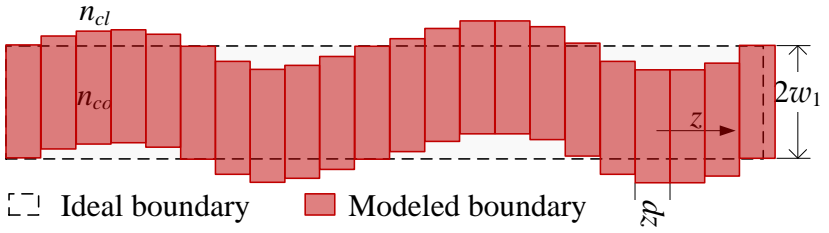

Fig. 1. Fiber dielectric waveguide with distorted core-cladding boundary.

the semi-analytical solutions including a validation of the accumulated mode coupling evolution with transmission length. Section VI presents a validation of the multi-section model for non-GD managed links by matching different analytical predictions for the statistics of the GDs, namely: standard deviation, probability density function, and cumulative distribution function. Section VII presents a validation of the multi-section model for GD managed links by matching the analytical predictions for standard deviation of the GDs. Conclusions are drawn in Section IV.

\section{COUPLED-MOdE THEORY FOR FEW-MOdE FIBERS}

The linear mode coupling in few-mode fibers is due to refractive-index inhomogeneities or small deviations of the core-cladding boundary caused by perturbations introduced during the fabrication process or by mechanical stresses imposed on the fiber in the field. Fig. 1 (a) shows a fiber dielectric waveguide with distorted core-cladding boundary. These imperfections cause the modes of the fiber to couple among each other. When exciting a pure mode at the fiber beginning, some of its power is transferred to other guided modes. This power transfer results in signal distortion because each guided mode travels at its own characteristic group velocity. Therefore, the equalization of the received signal must span over a time window that covers all the significant distortions undergone by a given information symbol.

Mode coupling may even be a desirable effect. The mode delay spread can be reduced by introducing a significant amount of distributed coupling among all guided modes which introduces a sufficiently strong averaging effect of the different mode group velocities, see section V. However, in mode delay compensated fiber links, mode coupling may or may not be desirable, as discussed in section VI.

\section{A. Coupled-Mode Equations}

The perturbations that couple the ideal modes of the ideal waveguide can be described by variations of the dielectric tensor. This is, the perturbed dielectric tensor $\left(\varepsilon_{p}\right)$ as a function of the space coordinates is written as:

$$
\varepsilon_{p}(x, y, z)=\varepsilon_{u}(x, y)+\Delta \varepsilon(x, y, z)
$$

where $\varepsilon_{u}(x, y)$ is the unperturbed part of the dielectric tensor, thereby invariant with the fiber longitudinal coordinate $z$, and $\Delta \varepsilon(x, y, z)$ represents the dielectric perturbation, which in the general case varies with all space coordinates. Eq. (1) can adequately describe the core-cladding perturbations in Fig. 1 (a).

If an arbitrary field of frequency $\omega$ is excited at $z=0$, the propagation of this field in the unperturbed dielectric waveguide can be expressed as a linear combination of the ideal modes:

$$
\mathbf{E}(x, y, z, t)=\sum A_{m}(z, t) \mathbf{E}_{m}(x, y) \mathrm{e}^{\mathrm{j}\left(w t-\beta_{m} z\right)}
$$

where $m$ is the mode index, $A_{m}(z, t)$ is the slowly varying mode field envelope, $\beta_{0 m}$ is the mode propagation constant at $\omega$, and $\mathbf{E}_{m}(x, y)$ is the electric field distribution.

In the presence of a dielectric perturbation $\Delta \varepsilon(x, y, z)$, the coupling between the ideal modes are described by the following coupled-mode equations [5], [7], [23]:

$$
\begin{array}{r}
{\left[\partial_{z}+\beta_{1 m} \partial_{t}-\mathrm{j} \frac{\beta_{2 m}}{2} \partial_{t}^{2}+\mathrm{j} \frac{\beta_{3 m}}{6} \partial_{t}^{3} \ldots\right] A_{m}(z, t)=} \\
-\mathrm{j} \sum_{n} C_{m, n}(z) A_{n}(z, t) e^{\mathrm{j}\left(\beta_{0 m}-\beta_{0 n}\right) z} \\
C_{m, n}(z)=\frac{\omega \varepsilon_{0}}{4} \iint_{-\infty}^{+\infty}[\Delta \varepsilon(x, y, z)] \mathbf{E}_{m}^{*} \cdot \mathbf{E}_{n} d x d y
\end{array}
$$

where $\beta_{l m}$ is the $l^{\text {th }}$ order coefficient of a Taylor series expansion of $\beta_{m}(\omega)$ centered at the carrier frequency $\omega . C_{m, n}$ are the coupling coefficients given by the area integral of the dot product of the electrical fields of mode $m$ and mode $n$, over the area where the permittivity perturbation $\Delta \varepsilon(x, y, z) \neq 0$.

For the general case, where $\Delta \varepsilon$ is varying continuously with $z$, so is $C_{m, n}$, the solution of the coupling operator in (1) can only be achieved using numerical methods, e.g. RungeKutta method. However, the usage of these methods is computationally inviable for simulation of long-haul transmission links. To overcome such limitation, we propose a model that discretizes the core-cladding fluctuations by dividing the fiber in multiple sections, each with a random displacement of the core center position constant along the section. In this case, the dielectric tensor is given by:

$$
\varepsilon_{p}(x, y, z)=\varepsilon_{r 0}(x+\delta x(z), y+\delta y(z), z)
$$

where $\delta x$ and $\delta y$ are the random displacement of the abscissa and ordinate coordinates, respectively. Fig. 1 (b) shows a diagram of the discretization of the core-cladding fluctuations given the proposed method. In this case, each section has constant coupling coefficients. Therefore, in theory it should be possible to find (semi-)analytical solutions for the coupling operator present in (3).

Assuming the fiber section length is much shorter than both the dispersion length $L_{D}=T_{0}^{2} /\left|\beta_{2 m}\right|$ and the walk-off length $L_{W}=T_{0} /\left|\beta_{1 m}-\beta_{1 n}\right|$, where $T_{0}$ is a measure of the pulse width, an approximate solution of (3) can be obtained by assuming the dispersive effects and linear coupling effects act independently. In the following, we will focus on finding a (semi-)analytical solution for the coupling operator, this is, we will be trying to solve:

$$
\partial_{z} A_{m}(z, t)=-\mathrm{j} \sum_{n} C_{m, n}(z) A_{n}(z, t) e^{\mathrm{j}\left(\beta_{0 m}-\beta_{0 n}\right) z}
$$


(a)

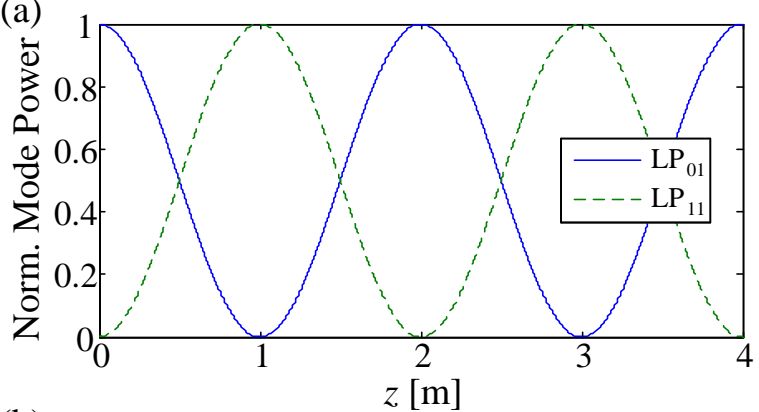

(b)

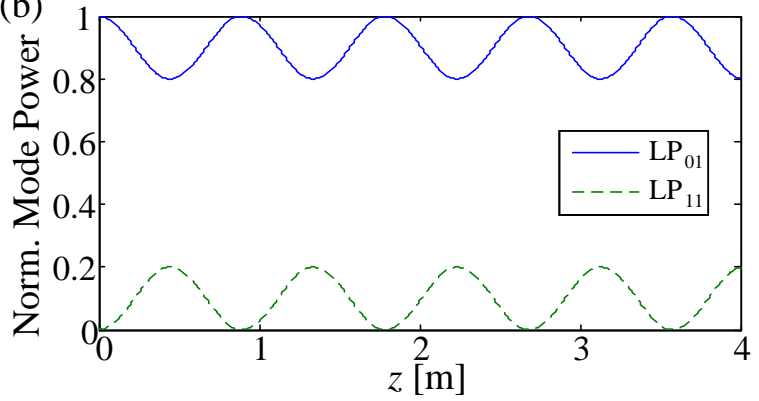

Fig. 2. Fiber dielectric waveguide with distorted core-cladding boundary.

\section{B. Coupled-Mode Equations Solution for Two-Mode Fibers}

The simple case of a two-mode fiber, where only the coupling between the $\mathrm{LP}_{01}$ mode $(m=1)$ and the $\mathrm{LP}_{11}$ mode $(n=2)$ is present, (6) can be solved analytically in each section [5]:

$$
\begin{gathered}
A_{1}(z)=e^{\mathrm{j}(\Delta \beta / 2) z}\left[\begin{array}{c}
\cos s z-\mathrm{j} \frac{\Delta \beta}{2} \frac{\sin s z}{s} \\
-\mathrm{j} \kappa \frac{\sin s z}{s}
\end{array}\right]^{T}\left[\begin{array}{l}
A_{1}(0) \\
A_{2}(0)
\end{array}\right] \\
A_{2}(z)=e^{-\mathrm{j}(\Delta \beta / 2) z}\left[\begin{array}{c}
-\mathrm{j} \kappa^{*} \frac{\sin s z}{s} \\
\cos s z+\mathrm{j} \frac{\Delta \beta}{2} \frac{\sin s z}{s}
\end{array}\right]^{T}\left[\begin{array}{l}
A_{1}(0) \\
A_{2}(0)
\end{array}\right]
\end{gathered}
$$

where $\Delta \beta=\beta_{01}-\beta_{02}, s^{2}=\kappa \kappa^{*}+(\Delta \beta / 2)^{2}$, and $\kappa=C_{12}=C_{21} *$. From (7) and (8), it can be concluded that the coupling strength depends on the relation between $|\kappa|^{2}$ and $\Delta \beta^{2}$.

Fig. 2 a) and Fig. 2 b) show the mode powers $\left|A_{1}\right|^{2}$ and $\left|A_{2}\right|^{2}$ as functions of the interaction distance $\mathrm{z}$, for $\Delta \beta=0$ and for $\Delta \beta=4|\kappa|$, respectively, with $\kappa=\pi / 2$. Fig. 2 shows that the coupling efficiency is $100 \%$ when the phase mismatch is zero, a full power swap happens for every $|\kappa| z$ odd multiple of $\pi / 2$. However, if the phase mismatch is different from zero the coupling is no longer the power coupling is incomplete, for $\Delta \beta=2|\kappa|$ the maximum coupling is $1 / 2$.

For higher number of modes, the dependence of the coupling strength on the phase mismatch and on the coupling coefficient should follow similar dependencies. Next section presents a solution method for higher number of modes.

\section{SEmi-Analytical SOlutions For HIGHER-ORDER MODE FIBERS}

For the simple case of a fiber with two modes, (6) can be easily solved by hand, however, this method becomes endless for higher number of modes. The use of a numerical method for the solution of (6), such as the Runge-Kutta-Fehlberg (RK45) method, is also not an option since it would be necessary to solve these equations for each fiber section with different fiber displacements, thus leading to computation times that are unaffordable in most applications. Therefore, an analytical approach is desirable.

Our approach starts by taking the Fourier Transform of (6) on $z$ to avoid the complex exponentials, obtaining:

$$
A_{m}\left(w_{z}\right)=-\frac{\mathrm{j}}{w_{z}} \sum_{n} C_{m, n} A_{n}\left(w_{z}-\Delta \beta_{m, n}\right)
$$

where $w_{z}$ is the spatial frequency and $\Delta \beta_{m, n}=\beta_{0 m}-\beta_{0 n}$. The system of equations (9) can be solved by substitution, thereby eliminating each $A_{p}$ from all the equations for all $p \neq m$, obtaining an equation for $A_{m}$ which can be written as:

$$
A_{m}\left(w_{z}\right) \cdot\left[a_{m, R}\left(\mathrm{j} w_{z}\right)^{R}+\ldots+a_{m, 0}\left(\mathrm{j} w_{z}\right)^{0}\right]=0
$$

where $R$ is equal to $2(M-1)$, with $M$ equal to the number of modes, and $a_{m, r}$ are functions of $\Delta \beta_{m n}$ and $C_{m n}$. The process described must be performed for $m=1, \ldots, M$ to obtain equations like (10) for each mode. Now, by applying the inverse Fourier transform to (10), a differential equation with constant coefficients is obtained which can be solved using the method of the characteristic polynomial, obtaining:

$$
A_{m}(z)=b_{m, 1} e^{s_{m, 1}}+\ldots+b_{m, R} e^{s_{m, R^{2}}}
$$

where $s_{m, r}$ are the characteristic polynomial roots and $b_{m, r}$ are constants that can be determined from the initial conditions at $z=0$ obtained by differentiating $(6),\left(d_{z}{ }^{i} A_{m}\right)_{z=0}$, and equating the results. Finally, the coefficients $b_{m, r}$ are the solutions of the linear equations system:

$$
\left[\begin{array}{ccc}
s_{m, 1} & \cdots & s_{m, N} \\
\vdots & \ddots & \vdots \\
s_{m, 1}^{2(M-1)} & \cdots & s_{m, N}^{2(M-1)}
\end{array}\right]\left[\begin{array}{c}
b_{m, 1} \\
\vdots \\
b_{m, R}
\end{array}\right]=\left[\begin{array}{c}
\left(d_{z}^{0} A_{m}\right)_{z=0} \\
\vdots \\
\left(d_{m}^{2(M-1)} A_{m}\right)_{z=0}
\end{array}\right]
$$

The solution method described is easily applied using a software tool with symbolic computation capability. We have used the Symbolic Math Toolbox from Matlab ${ }^{\circledR}$ to generate equations for $a_{m, r}$ and $\left(d_{z}{ }^{i} A_{m}\right)_{z=0}$ as function of $\Delta \beta_{m n}$ and $C_{m n}$. Finally, the derived equations can be written into a conventional text file and compiled using any programming language (we used a C-compiler). Afterwards, those equations can be evaluated allowing to find the roots of the polynomials in (10) and to solve the system of linear equations in (12).

In summary, instead of numerically solving a coupledsystem of $M$ differential equations (6), the method proposed requires the finding the roots of a $2(M-1)$ order polynomial, for which efficient and accurate algorithms are available, e.g. Bairstow's method [28], and the solution of a system of linear equations (12).

\section{A. Analytical expressions for the three-modes case}

The analytical expression for $a_{m, r}$ and $\left(d_{z}^{r} A_{m}\right)_{z=0}$ as a 
function of $\Delta \beta_{m, n}$ and $C_{m, n}$ for $M=3$ are given by equations (13) to (18), obtained executing the MATLAB code presented in Appendix. Replacing the $\Delta \beta_{m, n}$ and $C_{m, n}$ numeric values in the $a_{m, r}$ analytical equations, the $s_{m, r}$ values can be calculated using algorithms for the calculation of polynomial roots. Moreover, $\left(d_{z}{ }^{r} A_{m}\right)_{z=0}$ values are obtained replacing the $\Delta \beta_{m, n}$ and $C_{m, n}$ numeric values in the analytical formulas. Finally, the system of linear equations (12) can be numerically solved.

\section{B. Analytical expressions for more than three-modes}

The analytical expression for $a_{m, r}$ and $\left(d_{z}^{r} A_{m}\right)_{z=0}$ as a function of $\Delta \beta_{m, n}$ and $C_{m, n}$ for $M>3$ can be obtained executing Matlab code similar to that made available in the Appendix. However, the equations become too long to be printed here in full. The authors have made available the analytical equations for 6-modes and the respective MATLAB script for their derivation, at: http://doi.org/10.17036/researchdata.aston.ac.uk.00000206.

$$
\left[\begin{array}{l}
a_{1,0} \\
a_{1,1} \\
a_{1,2} \\
a_{1,3} \\
a_{1,4}
\end{array}\right]=\left[\begin{array}{c}
1 \\
-2 \mathrm{j} \Delta \beta_{12}-\mathrm{j} \Delta \beta_{13} \\
C_{12}{ }^{2}-\Delta \beta_{12}{ }^{2}-2 \Delta \beta_{12} \Delta \beta_{13}+C_{13}{ }^{2}+C_{23}{ }^{2} \\
\mathrm{j}\left(\begin{array}{c}
\Delta \beta_{12}{ }^{2} \Delta \beta_{13}-\Delta \beta_{12} C_{12}{ }^{2}-2 \Delta \beta_{12} C_{13}{ }^{2} \\
-\Delta \beta_{13} C_{12}{ }^{2}-\Delta \beta_{12} C_{23}{ }^{2}-2 C_{12} C_{13} C_{23}
\end{array}\right) \\
-\Delta \beta_{12}\left(\Delta \beta_{12} C_{13}{ }^{2}+\Delta \beta_{13} C_{12}{ }^{2}+2 C_{12} C_{13} C_{23}\right)
\end{array}\right]
$$

$$
\left[\begin{array}{l}
a_{2,0} \\
a_{2,1} \\
a_{2,2} \\
a_{2,3} \\
a_{2,4}
\end{array}\right]=\left[\begin{array}{c}
-1 \\
-3 \mathrm{j} \Delta \beta_{12}+\mathrm{j} \Delta \beta_{13} \\
\mathrm{j}\left(\begin{array}{c}
-\Delta \beta_{12}{ }^{2} \Delta \beta_{13}-2 \Delta \beta_{12} \Delta \beta_{13}-C_{12}{ }^{2}-C_{13}{ }^{2}-C_{12}{ }^{2}-\Delta \beta_{12} C_{13}{ }^{2}+\Delta \beta_{13} C_{12}{ }^{2} \\
-2 \Delta \beta_{12} C_{23}{ }^{2}+\Delta \beta_{12}{ }^{3}+2 C_{12} C_{13} C_{23}
\end{array}\right) \\
\Delta \beta_{12}\left(\Delta \beta_{12} C_{12}{ }^{2}-\Delta \beta_{13} C_{12}{ }^{2}+\Delta \beta_{12} C_{23}{ }^{2}-2 C_{12} C_{13} C_{23}\right)
\end{array}\right]
$$$$
\left[\begin{array}{l}
a_{3,0} \\
a_{3,1} \\
a_{3,2} \\
a_{3,3} \\
a_{3,4}
\end{array}\right]=\left[\begin{array}{c}
-1 \\
\mathrm{j} \Delta \beta_{12}-3 \mathrm{j} \Delta \beta_{13} \\
\mathrm{j}\left(\begin{array}{c}
-\Delta \beta_{12} \Delta \beta_{13}{ }^{2}+\Delta \beta_{12} k_{13}{ }^{2}-\Delta \beta_{13} C_{12}{ }^{2} \\
-2 \Delta \beta_{13} C_{13}{ }^{2}-2 \Delta \beta_{13} C_{23}{ }^{2}+\Delta \beta_{13}{ }^{3}+2 C_{12} C_{13} C_{23}
\end{array}\right) \\
-\Delta \beta_{13}\left(C_{13}{ }^{2}\left(\Delta \beta_{12}-\Delta \beta_{13}\right)-2 \Delta \beta_{13} C_{23}+2 C_{12} C_{13} C_{23}\right)
\end{array}\right]
$$

$$
\left[\begin{array}{l}
d_{z}^{0} A_{1} \\
d_{z}^{1} A_{1} \\
d_{z}^{2} A_{1} \\
d_{z}^{3} A_{1}
\end{array}\right]_{z=0}=\left[\begin{array}{c}
A_{1} \\
-\mathrm{j} C_{12} A_{2}-\mathrm{j} C_{13} A_{3} \\
\left\{\begin{array}{c}
-C_{12}\left(C_{12} A_{1}+C_{23} A_{3}\right)-C_{13}\left(C_{13} A_{1}+C_{23} A_{2}\right) \\
+d B_{12} C_{12} A_{2}+d B_{13} C_{13} A_{3}
\end{array}\right\} \\
\mathrm{j}\left\{\begin{array}{c}
-C_{12}\left(C_{12} A_{2}+C_{13} A_{3}\right) \\
-C_{12}\left(\begin{array}{c}
-C_{23}\left(C_{13} A_{1}+C_{23} A_{2}\right) \\
-d B_{12} C_{12} A_{1}+d B_{23} C_{23} A_{3}
\end{array}\right) \\
-C_{13}\left(\begin{array}{l}
-C_{13}\left(C_{12} A_{2}+C_{13} A_{3}\right) \\
-C_{23}\left(C_{12} A_{1}+C_{23} A_{3}\right) \\
-d B_{13} C_{13} A_{1}-d B_{23} C_{23} A_{2}
\end{array}\right) \\
-2 d B_{12} C_{12}\left(C_{12} A_{1}+C_{23} A_{3}\right) \\
-2 d B_{13} C_{13}\left(C_{13} A_{1}+C_{23} A_{2}\right) \\
+d B_{12}{ }^{2} C_{12} A_{2}+d B_{13}{ }^{2} C_{13} A_{3}
\end{array}\right\}
\end{array}\right\}
$$

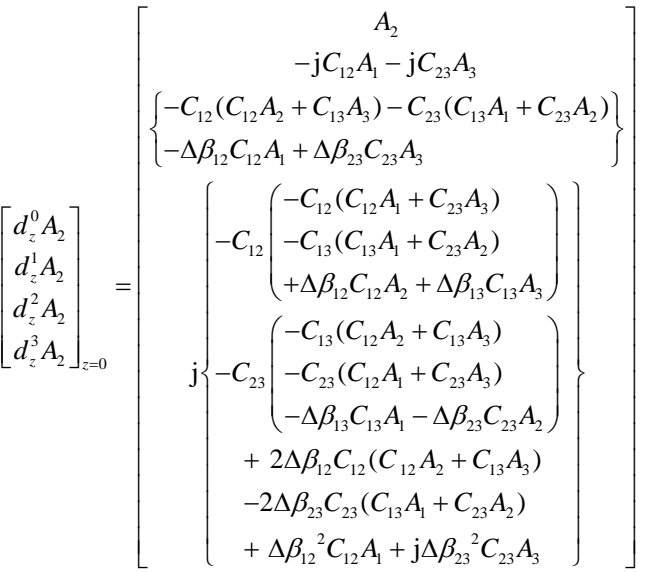

$$
\begin{aligned}
& A_{3} \\
& -\mathrm{j} C_{13} A_{1}-\mathrm{j} C_{23} A_{2} \\
& \left\{-C_{13}\left(C_{12} A_{2}+C_{13} A_{3}\right)-C_{23}\left(C_{12} A_{1}+C_{23} A_{3}\right)\right. \\
& \left\{-\Delta \beta_{13} C_{13} A_{1}-\Delta \beta_{23} C_{23} A_{2}\right. \\
& {\left[\begin{array}{l}
d_{z}^{0} A_{3} \\
d_{z}^{1} A_{3} \\
d_{z}^{2} A_{3} \\
d_{z}^{3} A_{3}
\end{array}\right]_{z=0}=} \\
& -C_{13}\left(\begin{array}{l}
-C_{12}\left(C_{12} A_{1}+C_{23} A_{3}\right) \\
-C_{13}\left(C_{13} A_{1}+C_{23} A_{2}\right) \\
+\Delta \beta_{12} C_{12} A_{2}+\Delta \beta_{13} C_{13} A_{3}
\end{array}\right) \\
& \mathrm{j}\left\{-C_{23}\left(\begin{array}{l}
-C_{12}\left(C_{12} A_{2}+C_{13} A_{3}\right) \\
-C_{23}\left(C_{13} A_{1}+C_{23} A_{2}\right) \\
-\Delta \beta_{12} C_{12} A_{1}+\Delta \beta_{23} C_{23} A_{3}
\end{array}\right)\right. \\
& +2 \Delta \beta_{13} C_{13}\left(C_{12} A_{2}+C_{13} A_{3}\right) \\
& +2 \Delta \beta_{23} C_{23}\left(C_{12} A_{1}+C_{23} A_{3}\right) \\
& +\Delta \beta_{13}{ }^{2} C_{13} A_{1}+\mathrm{j} \Delta \beta_{23}{ }^{2} C_{23} A_{2}
\end{aligned}
$$

\section{Algorithm Complexity}

The RK45 method requires a step-size of a fraction of the beat-length between the two mode-groups most farther apart, which can easily be of the order of a millimeter or less [11]. In this way, to resolve a one millimeter beat-length, more than $10^{4}$ and $10^{6}$ steps are required for a transmission length of 1 and 100 meters, respectively. Each RK45 step requires six evaluations of a system of $M$ equations (6), each equation with $2(M-1)$ multiplications, thus totalizing $12 M(M-1)$ multiplication operations per step.

The semi-analytical method proposed uses the Bairstow's method to find the roots of $M$ polynomials of order 2(M-1). This method consists on the progressive division of the original polynomial by quadratic polynomials while adjusting the coefficients of the later. Thus, the method requires $(M-1)$ polynomial divisions of progressively lower complexity. Assuming the number of multiplications required to be the product of the number of terms of the polynomials involved, the $i^{\text {th }}$-division requires $[(2(M-1)+1)-2(i-1)](2+1)$, adding up to $3\left(M^{2}-1\right)$ multiplication operations. Finally, this figure must be multiplied by the number of iterations for coefficients adjustment, which we cap to be lower than 100, and observed that in general only 20 repetitions were required. Thus, the total complexity is on the order of $60 M\left(M^{2}-1\right)$.

Finally, the proposed semi-analytical method reduces the number of multiplications required by a factor from 280 to 28000 when transmitting over 1 to 100 meters, for $M=6$. These factors agree with the observed simulation times. 


\section{Single-SECtion Modelling}

In this section, the semi-analytical solutions of section III are validated using the Runge-Kutta-Fehlberg (RK45) method [29]. The fiber considered for guides six linearly polarized (LP) modes: $\mathrm{LP}_{01}, \mathrm{LP}_{11 \mathrm{a}}, \mathrm{LP}_{11 \mathrm{~b}}, \mathrm{LP}_{21 \mathrm{a}}, \mathrm{LP}_{21 \mathrm{~b}}$, and $\mathrm{LP}_{02}$. It has a relative index gradient at the core-cladding interface $4.5 \times 10^{-3}$ and a core radius $\left(w_{1}\right)$ of $12.83 \mu \mathrm{m}$, optimization details [27]. Table I shows the fiber characteristics at $1550 \mathrm{~nm}$ (for the sake of clarity, the modes were numbered from one to six). Fig. 3 depicts the amplitude of $C_{m, n}$ as a function of the fiber displacement vector for a radial displacement from 0 to $0.3 \cdot w_{1}$. Note that, the coupling coefficients were found to be real and symmetric has concluded in [23], therefore only $C_{m, n}$ with $n>m$ are shown. From Fig. 3, the pairs of modes with higher coupling strength can be identified, and it can be verified that the coupling between symmetric modes $\left(\mathrm{LP}_{01}\right.$, for example) and anti-symmetric modes ( $\mathrm{LP}_{11}$, for example) requires a non-symmetrical perturbation. The surfaces shown in Fig. 3 allow the rapid calculation of the coupling coefficients $C_{m, n}$ using interpolation for a random displacement, as required for integration in a modified splitstep Fourier method (SSMF).

In the following, the semi-analytical (SA) solutions are compared to the numerical solutions obtained using the RK45 method. The variable-step width of the RK45 method is specified considering a relative error tolerance of $10^{-6}$ and an absolute error tolerance of $10^{-9}$. Fig. 4 shows the overlap of the modal powers given by the SA solutions and the modal powers given by the numerical method as a function of $z$, considering a fiber core displacement of $\rho_{d}=0.08 \cdot w_{1}$ and $\varphi_{d}=\pi / 3$, for an even power distribution between the modes at the input. A very good agreement between the SA and numerical solutions can be noticed in Fig. 4 inset which zooms in the mode power evolution around $0.8 \mathrm{~m}$. Similar agreement is obtained for different input conditions.

To have a better measure of the error magnitude, the mean squared error (MSE) between the semi-analytical (SA) results and the numerical (NUM) results is calculated for each mode, $M S E_{m}$, given by:

$$
M S E_{m}=\frac{1}{N} \sum_{n=1}^{N}\left|A_{m}^{S A}\left(z_{n}\right)-A_{m}^{N U M}\left(z_{n}\right)\right|^{2}
$$

where $A^{S A}{ }_{m}$ is the SA mode amplitude solution, $A^{N U M}{ }_{m}$ is the numerical mode amplitude solution, and $z_{n}$ are the discrete points considered in a specific fiber length. $M S E_{m}$ has been calculated considering $10^{5}$ discrete points equally spaced along a fiber with $1 \mathrm{~m}$, considering $\rho_{d}$ varying 0 and $0.08 \cdot \mathrm{w}_{1}$ (1000 points equally spaced), and $\varphi_{d}$ varying from $-\pi$ to $\pi$ (1000 points equally spaced). In all the cases tested the $M S E_{m}$ was always of the order of magnitude of the RK45 absolute tolerance, as verified by repeating the error calculation for different tolerance values. Therefore, it can be concluded that the semi-analytical method proposed provides an accurate estimative of the linear mode coupling taking place along a FMF. More importantly, using the semi-analytical method the computation time required to calculate the linear coupling
TABLE I

FIBER PROPERTIES AT $1550 \mathrm{~nm}$.

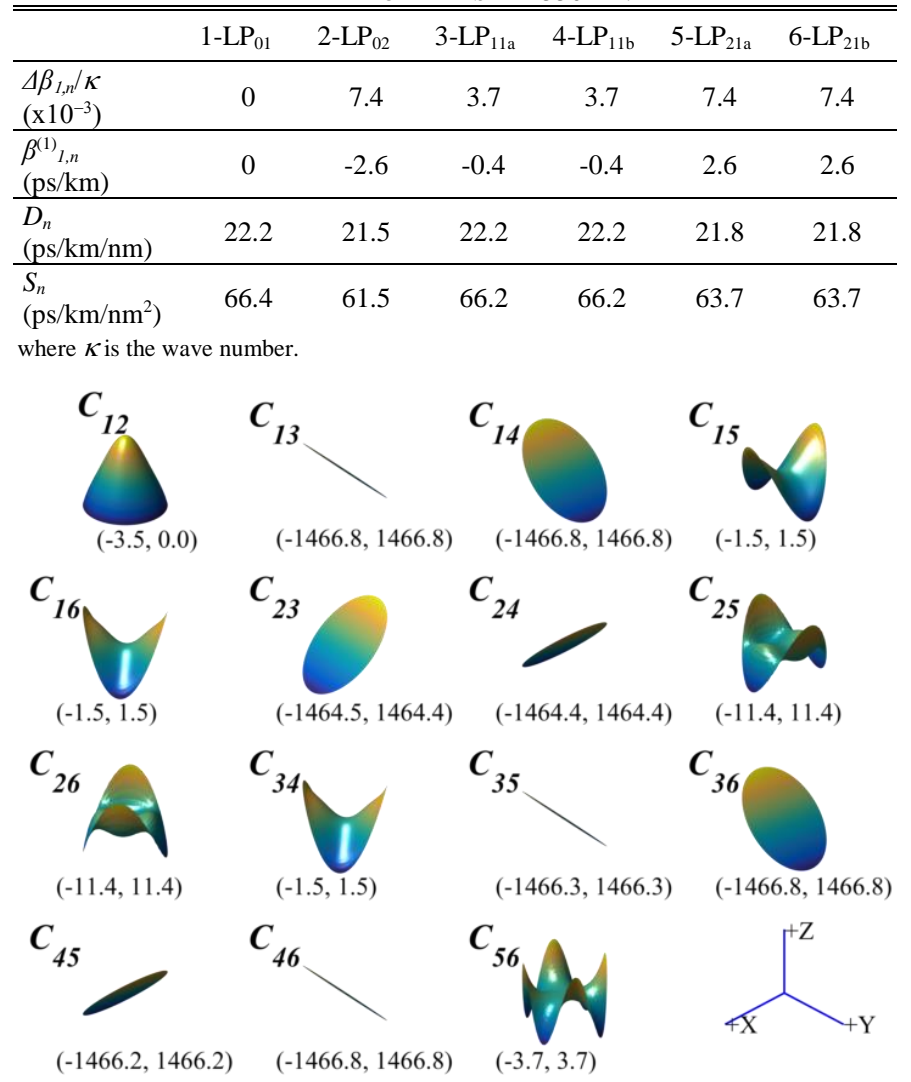

Fig. 3. $C_{m, n}$ as a function of the fiber displacement vector, minimum and maximum values as ( $\min , \max )$.

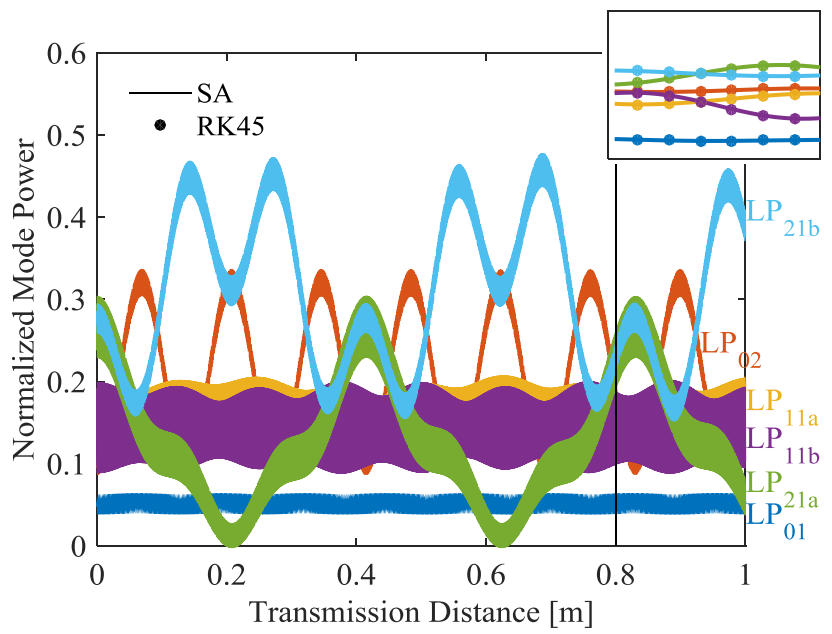

Fig. 4. Normalized mode power as a function of the fiber length, for uneven power distribution at the fiber input. The subscripts SA and RK45 were used for semi-analytical and numerical solutions, respectively. The inset shows the excellent agreement between SA and RK45 around $0.8 \mathrm{~m}$.

along a fiber with a few meters is reduced by three orders of magnitude compared to the RK45 method which required tens of seconds executing on a standard personal computer operating at $2.8 \mathrm{GHz}$.

In conclusion, the semi-analytical solutions obtained enable a time efficient and accurate computation of the linear coupling occurring along the fiber length. They are therefore a valuable alternative to the numerical solution, which would not be practical due to computation time constraints. 


\section{Multi-Section Modelling}

We propose a multi-section model were the coupling strength is set using a given radial displacement and a uniformly distributed azimuthal displacement for each section. The radial displacement to be used depends not only on the target coupling strength but also on the fiber step length to be used. But first we quantitatively define the mode coupling strength and present its dependence on the radial displacement.

\section{A. Setting Mode Coupling Strength and Correlation Length}

The statistical nature of polarization mode dispersion (PMD) in SMFs is mainly determined by the correlation length, which is defined in terms of fiber mode coupling. In SMFs, the mode coupling is easily defined as there is only two polarizations, and the $L_{c}$ is defined as the length for which the average power in the orthogonal polarization is within $e^{-2}$ of the power in the launching polarization. In FMFs, the mode coupling strength can be quantified as the ratio between the average power in all the other orthogonal modes and average power remaining in the launching mode, after a certain distance. Thus, there are as many coupling strength values and $L_{c}$ as the number of modes. Inevitable, the fiber mode $m$ showing higher coupling strength will set an important reference for the study of the mode group-delay statistics. Finally, the mode coupling strength definition for FMFs is:

$$
X T_{m}=\sum_{v \neq \mathrm{m}}\left(P_{v} / P_{m}\right),
$$

where $P_{v}$ is the power of mode $v$, after a given fiber segment under test, when only the $m$ mode was launched, where $m$ is the mode that shows higher coupling strength. In the FMF case, we generalize $L_{c}$ for mode $m$ as the length for which $\left(P_{m}-\sum_{v \neq \mathrm{m}} P_{v}\right)=e^{-2}$, this is $X T_{m}=\left[e^{2}-1\right] /\left[e^{2}+1\right](-1.18 \mathrm{~dB})$.

In our multi-step model a given amount of coupling is set by selecting a fixed amount of radial displacement and selecting a random azimuth displacement given by a uniform distribution. In this way, the proposed model introduces a random amount of crosstalk per step that in average approximates the desired level. Fig. 5 shows the mode coupling strength averaged over the azimuth displacement, as a function of the normalized radial displacement, for a 6 LP mode fiber presented in section IV. Note that, coupling strengths are calculated considering degenerate modes such as $\mathrm{LP}_{11 \mathrm{a}}$ and $\mathrm{LP}_{11 \mathrm{~b}}$ as one mode, e.g. $X T_{\mathrm{LP} 11 \mathrm{a}, \mathrm{b}}$ equals to $\sum_{v \neq \mathrm{L} \mathrm{P}_{11 \mathrm{a}, \mathrm{b}}}\left\{P_{v} /\left(P_{\mathrm{LP}_{11 \mathrm{a}}}+P_{\mathrm{LP}_{11 \mathrm{~b}}}\right)\right\}$. In Fig. 5, the mode coupling strength only depends significantly on the mode being considered for displacements higher than $1 \%$. Such higher coupling for $\mathrm{LP}_{02}$ and $\mathrm{LP}_{21}$ can be explained noting they belong to the same LP mode group. Moreover, $X T_{\mathrm{LP}_{21}} \leq X T_{\mathrm{LP}_{02}}$ for any displacement in Fig. 5 because any power launched in $\mathrm{LP}_{21 \mathrm{a}}$ couples preferentially with $\mathrm{LP}_{21 \mathrm{~b}}$ (and vice-versa) and in the second place to $\mathrm{LP}_{02}$. Given the higher values of $X T_{\mathrm{LP}_{02}}$, we define $L_{c}$ for this mode. Note that $X T_{m}$ values above $10 \mathrm{~dB}$ mean that almost all power launched in mode $m$ has been transferred to other modes.

In the literature, the mode coupling values of fabricated

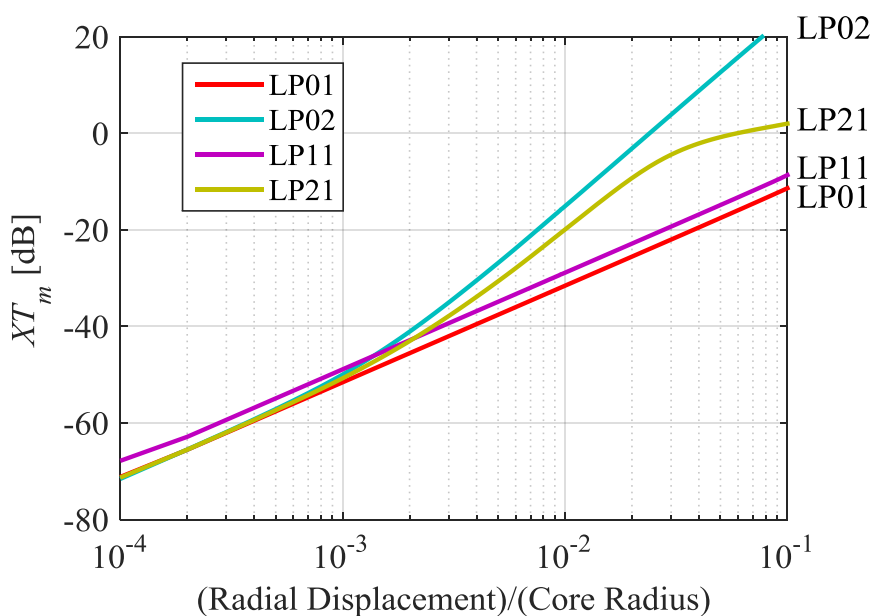

Fig. 5. $X T_{m}$ averaged over the azimuth displacement as a function of the radial displacement.

FMFs range from $-50 \mathrm{~dB} / 100 \mathrm{~m}$ to $-40 \mathrm{~dB} / 100 \mathrm{~m}$ for fibers with step-index or graded-index profiles [18], [19], going up to $-28 \mathrm{~dB} / 100 \mathrm{~m}$ for coupled multi-core fibers [20] and $-7 \mathrm{~dB} / 100 \mathrm{~m}$ for fibers with ring-index profiles [21].

\section{B. Mode Coupling Accumulation over Transmission Length}

In a multi-section model, the mode coupling accumulates section after section in such a way that in average should follow the same continuous growing function that was first derived to describe the accumulation of polarization coupling in polarization-maintaining fibers [30]:

$$
X T=\tanh (h z)
$$

where $h$ is the mode coupling parameter (measured in $\mathrm{m}^{-1}$ units) and $z$ is the fiber length.

To validate our multi-section model, we have run 10,000 transmission simulations considering the 6-mode fiber presented in section IV. Fig. 6 shows the average $X T_{\mathrm{LP}_{02}}$ as a function of the fiber length $(L)$ from $10 \mathrm{~m}$ to $1000 \mathrm{~km}$, considering a fiber section of $10 \mathrm{~m}$ and different values of coupling strength. Note that the dashed lines in Fig. 6 represent the evolution predicted for (21) using the respective $h$ coefficient. A very good agreement between the proposed multi-section model and (21) is noticeable. Furthermore, similar matches were obtained for other section sizes and respective radial displacements.

The executable code to generate the coupling matrices for different strengths as presented in Fig. 6 are available at: http://doi.org/10.17036/researchdata.aston.ac.uk.00000206.

\section{Polarization Mode Coupling}

After a few meters, the two nearly degenerate polarization modes of each spatial mode strongly couple to each other and the FMF enters the polarization coupling state [7], [23]. Such propagation mode coupling can be described by a block diagonal matrix with a sequence of $M / 2$ submatrices along the diagonal. Each of these $2 \times 2$ random unitary submatrices is a PMD transfer matrix [7].

The full coupling matrix for the $i^{\text {th }}$-section is modelled as the product of two matrices: one block diagonal matrix 


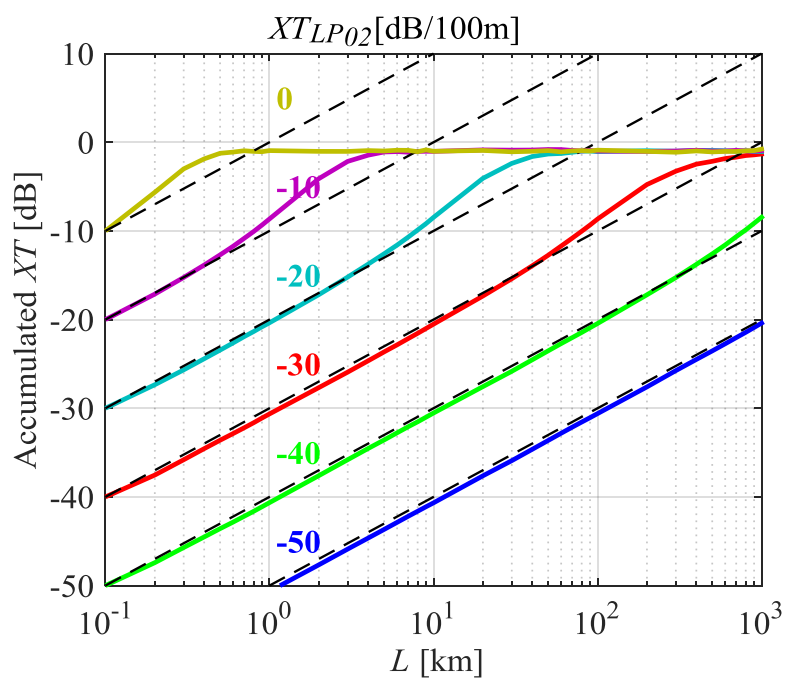

Fig. 6. Accumulated XT as a function of the fiber length, for different coupling strength, averaged over 10,000 runs.

describing the polarization mode coupling, and one matrix describing finite inter- and intra-mode group coupling (as described in Section III, IV, V-A and V-B). This approach follows a similar reasoning to that in [7] and [11] to deal with coupling processes having different correlation lengths.

\section{GD Statistics In NON-DELAY-MANAGED LinKS}

In previous work [31], it has been shown that the approach of considering principal states of polarization (PSPs) with well-defined GDs in SMFs, can be extended to FMFs. In FMFs, the coupled modes having well defined GDs are called principal modes (PMs). In both cases the statistics of the GDs are dependent on the linear coupling strength, thus the correlation length $L_{c}$. The coupling regimes may then be broadly defined as strong coupling when $L \gg L_{c}$, weak coupling when $L<<L_{c}$, and intermediate coupling otherwise. In the FMF case, the statistical properties of the GDs are well known for the two extreme regimes [6]-[10]. In the weak coupling regime, the GD spread grows linearly with distance and in the strong coupling regime grows with the square root of the distance. In the intermediate coupling regime, we have shown in [26] through simulation that the GDs statistics in SMFs can be extended to FMFs, at least for fibers guiding 3 LP modes. At the same time, the complete analytical derivation of such extension was presented in [32].

The temporal spread of propagating pulse is determined by the modal dispersion (MD) vector $\tau$, as defined for a generalized $\left(M^{2}-1\right)$-dimensional Stokes space in [9] $(M$ modes). Knowledge of the MD vector allows the extraction of the PMs and respective GDs as explained in [9]. Moreover, the square modulus of the MD vector is proportional to the sum of the GDs $\tau_{i}$ (with $\sum \tau_{i}=0$ ) [9]:

$$
\|\tau\|^{2}=M \sum_{i=1}^{M} \tau_{i}^{2}
$$

In this way, it can be noted that $\|\boldsymbol{\tau}\| / M$ is the standard deviation of the GD vector $\left[\tau_{1}, \tau_{2}, \ldots \tau_{M}\right], \sigma_{g d}$. The MD vector has been used to explicitly determine the delay spread $T$ in two limiting cases: one in which the PMs change rapidly across the signal bandwidth, and one in which the bandwidth of the PMs is much larger than the signal bandwidth. In the first case, $T$ is a deterministic quantity and determined by $\sigma_{g d}$, $T^{2}=E\left\{\|\boldsymbol{\tau}\|^{2}\right\} / M^{2}=E\left\{\sigma_{g d^{2}}{ }^{2}\right\} \quad[10]$, where $E\{\}$ denotes expectation. In the latter case, $T$ is a random quantity given by $\max _{i}\left\{\tau_{i}\right\}-\min _{i}\left\{\tau_{i}\right\}$ [6], [9], determined by the GD PDF.

In the following, we review the known MD statistics and use them to validate the multi-section model proposed in section $\mathrm{V}$ for a fiber guiding $6 \mathrm{LP}$ modes despite the different coupling strengths between different pairs of modes belonging to different mode groups. The FMF presented in section IV is considered again, the modal and chromatic dispersion values are given in Table I. The fiber presents a DMD of $5.19 \mathrm{ps} / \mathrm{km}$ and we assumed zero DMD between degenerate LP modes and between orthogonal polarizations. As explained in section V-B, the polarization mode coupling is considered in each section using a block diagonal matrix. Regarding the coupling matrix describing finite inter- and intra-mode group coupling, the $X T_{\mathrm{LP}_{02}}$ value was varied from -50 to $0 \mathrm{~dB} / 100 \mathrm{~m}$ by using a given radial displacement and a uniformly distributed azimuthal displacement for each section (see Fig. 5), assuming a section length of $10 \mathrm{~m}$. This range fully covers the range of coupling values presented in the literature [18]-[21]. Finally, the GDs of the PMs are the eigenvalues of the semi-analytically simulated transmission matrix. Note that the simulated transmission matrix must be compensated for chromatic dispersion as introduced by (3).

\section{A. GD Standard Deviation and Intensity Impulse Response}

Knowledge of the modulus of the MD vector $\|\boldsymbol{\tau}\|$ allows to determine the standard deviation of the GD vector $\left[\tau_{1}, \tau_{2}, \ldots \tau_{M}\right] \sigma_{g d}$, since $\sigma_{g d}=\|\boldsymbol{\tau}\| / M . E\left\{\|\boldsymbol{\tau}(z)\|^{2}\right\}$ can be found by integration of two deterministic differential equations $(z$ dependence is omitted) [12], [32]:

$$
\begin{array}{r}
\partial_{z} E\left\{\|\boldsymbol{\tau}\|^{2}\right\}=E\left\{2 \partial_{\omega} \boldsymbol{\beta} \boldsymbol{\tau}\right\}=2 \partial_{\omega} \boldsymbol{\beta} E\{\boldsymbol{\tau}\} \\
\partial_{z} E\{\boldsymbol{\tau}\}=\partial_{\omega} \boldsymbol{\beta}-1 / L_{c} E\{\boldsymbol{\tau}\}
\end{array}
$$

where $\partial_{\omega} \boldsymbol{\beta}$ term represents the uncoupled GDs per unit length and $L_{c}$ is the correlation length characteristic of the fiber, considering the same $L_{c}$ for all groups of modes.

For non-DMD-managed spans (this is, $\partial_{\omega} \boldsymbol{\beta}$ constant), $E\left\{\|\boldsymbol{\tau}(z)\|^{2}\right\}$ can be found through analytical integration of (23), and is given by [12], [32]:

$$
E\left\{\|\boldsymbol{\tau}\|^{2}\right\}=2\left\|\partial_{\omega} \boldsymbol{\beta}\right\|^{2} L_{c}^{2}\left(e^{-z / L_{c}}+z / L_{c}-1\right)
$$

Equation (24) was proposed and validated by simulation in [26] for fibers guiding 3 LP modes, and at the same time its analytical derivation being presented in [32].

Fig. 7 shows the standard deviation of the GD vector $\left(\left[\tau_{1}, \tau_{2}, \ldots \tau_{12}\right]\right)$ as a function of distance up to $1,000 \mathrm{~km}$, obtained by averaging over 6,000 different realizations of lateral offsets giving rise to a given $X T_{\mathrm{LP}_{02}}$ value. These results were obtained using the fiber presented in Table I, treating the polarization mode coupling as described in 


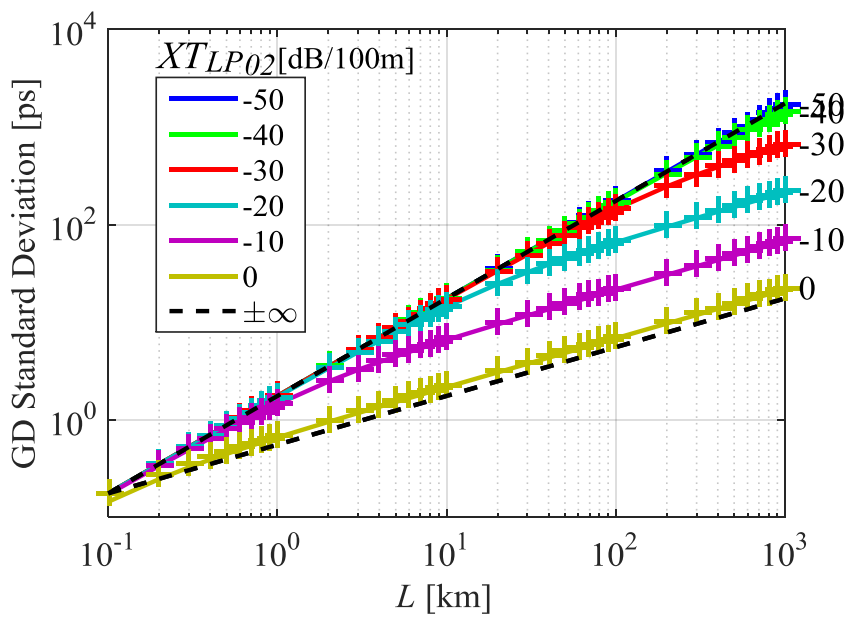

Fig. 7. Standard deviation of the GDs of the PMs as a function of transmission distance showing simulation results (markers) and analytical results (solid lines).

section V-B. Fig. 7 shows a good agreement between simulation and (24), for any coupling value studied and for any distance up to $1,000 \mathrm{~km}$ (even $10,000 \mathrm{~km}$ has further results shown). Similar agreement between (24) and simulation results has been presented in Fig. 3 of [12]. This provides mutual validation of (24) and the proposed multisection model proposed. In Fig. 7, for coupling values ranging from -50 to $-40 \mathrm{~dB} / 100 \mathrm{~m}, \sigma_{g d}$ scales approximately linearly with distance. But, at $-40 \mathrm{~dB} / 100 \mathrm{~m}$ the deviation from linear growth is already noticeable around $1,000 \mathrm{~km}$, thus even with such a low coupling, the FMF is operating in intermediate coupling regime. Increasing $X T_{\mathrm{LP}_{02}}, \sigma_{g d}$ gradually converges to the strong coupling regime. However, even for a $X T_{\mathrm{LP}_{02}}$ equal to $-7.01 \mathrm{~dB} / 100 \mathrm{~m}$ (the highest value found in literature [21]), the fiber is still not well modelled by random unitary matrices every $100 \mathrm{~m}$, it would underestimate $\sigma_{g d}$ by a factor of 2.76 .

For FMFs where the PMs change rapidly across the signal bandwidth, MD can be conviniently characterized by exciting each spatial channel (one at a time) with a short optical pulse and measuring the received intensities in each of the output spatial channels. Such process leads to $M \times M$ intensity waveforms, whose sum $I(t)$ has been used to assess the signal delay spread caused by MD [10], [12]. For strong modecoupling and typical MD values, it has been shown that $I(t)=r(t) * I_{0}(t)$ [10], where $*$ represents convolution, $I_{0}(t)$ is the launching signal intensity waveform, and $r(t)$ is FMF's intensity impulse response (IIR). Also in [10], it was shown theoretically and experimentally that $r(t)$ is a Gaussian function with variance equal to $T^{2}=E\left\{\|\boldsymbol{\tau}\|^{2}\right\} / M^{2}=E\left\{\sigma_{g d}{ }^{2}\right\}$, thus:

$$
r(t)=\frac{1}{\sqrt{2 \pi T^{2}}} \exp \left(\frac{-t^{2}}{2 T^{2}}\right)
$$

Equation (25) is valid as long as the correlation bandwidth $\left(B_{M D}=1 / 2 \pi T\right)$ of the fiber transfer matrix is much smaller than the channel bandwidth $(B \sim$ tens of GHz). Fig. 8 shows the mode-averaged intensity waveform for $M=12$ modes after transmission of a Nyquist signal $I_{0}(\mathrm{t})=\sqrt{\mathrm{B}} \sin (\pi B t) /(\pi B t)$, with $B=20 \mathrm{GHz}$, over a $1000 \mathrm{~km}$ link with coupling values ranging from -30 to $0 \mathrm{~dB} / 100 \mathrm{~m}$.

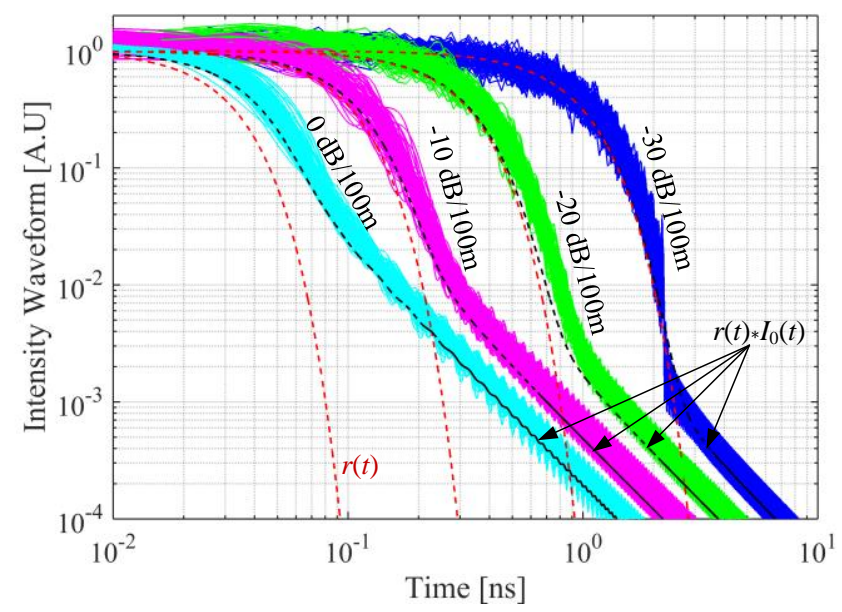

Fig. 8. Mode-averaged intensity waveform for different coupling values after transmission of a Nyquist signal. Simulation results, $r(t) * I_{0}(t)$ and $r(t)$ plotted using colored full lines, black dashed lines and red dashed lines, respectively.

Simulation results, $r(t) * I_{0}(t)$ waveform, and $r(t)$ IIR are plotted using colored full lines, black dashed lines, and red dashed lines, respectively. Fig. 8 displays simulation results for 100 different fiber realizations for each $X T_{\mathrm{LP} 02}$ value. All the waveforms were normalized so that their peak value is one. Fig. 8 shows an excellent agreement between simulations and theory (experimentally validated) as obtained in [10]. Note that the deviations from theory reduce as the coupling strenght increases and the PMs bandwidth decreases. Finally, further results show that the deviation of $T(z)$ from theory is in agreement with the theory in [10].

\section{B. GD Probability Density Function and Maximum GD Spread}

The probability density function (PDF) of the GDs has been derived analytically for strong coupling [6] where the coupling matrix can be described as a Gaussian unitary ensemble. The ordered joint pdf of the eigenvalues $\left(\tau_{i}\right)$ of a $M \times M$ Gaussian unitary ensemble with zero trace $\left(\sum \tau_{i}=0\right)$ is:

$$
p_{M}\left(\tau_{1}, \ldots, \tau_{M-1}\right)=\rho_{M} \prod_{M \geq i>j>0}\left(\tau_{i}-\tau_{j}\right)^{2} e^{\left(-\sum_{i=1}^{M} \tau_{i}^{2}\right)}
$$

with order constrain $\tau_{1} \leq \tau_{2} \leq \ldots \leq \tau_{M}$ and where the constant $\rho_{M}$ is defined by requiring (26) to integrate to unity. The unordered joint PDF is just $1 / M$ ! of (26) but without the order constraint. In this way, the marginal PDF of $\tau$ is can be obtained by integrating over $\tau_{2}, \ldots, \tau_{M-1}$ :

$$
p_{M \tau}(\tau)=\frac{1}{M !} \int_{-\infty}^{+\infty} \ldots \int_{-\infty}^{+\infty} p_{M}\left(\tau, \tau_{2}, \ldots, \tau_{M-1}\right) d \tau_{2} \ldots d \tau_{M-1}
$$

Analytical solutions of (27) can be find in [6]-[7] for any $M$.

Fig. 9 shows the PDF of the ordered GDs ( $\tau_{m}$, $\left.\tau_{1} \leq \tau_{2} \leq \ldots \leq \tau_{6}\right)$ obtained for 6000 different fiber matrix realizations, normalized by the $\sigma_{g d}$, after $1000 \mathrm{~km}$ for two different coupling values, overlapped with the analytical joint PDF (thin black line) derived for the strong coupling regime (27). Note that the normalization factor $\left(\sigma_{g d}\right)$ depends on the $X T_{\mathrm{LP}_{02}}\left(L_{c}\right)$ value, see (24). Exceptionally, these results were obtained for single-polarization to facilitate the visualization 


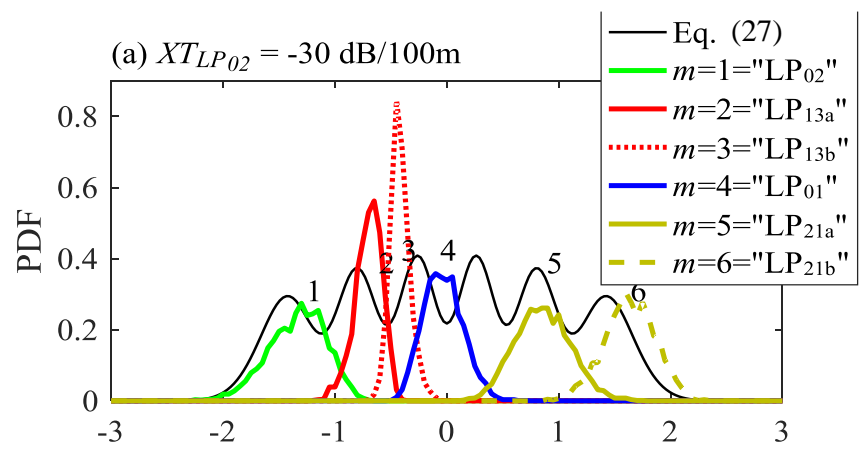

(b) $X T_{L P_{02}}=-20 \mathrm{~dB} / 100 \mathrm{~m}$

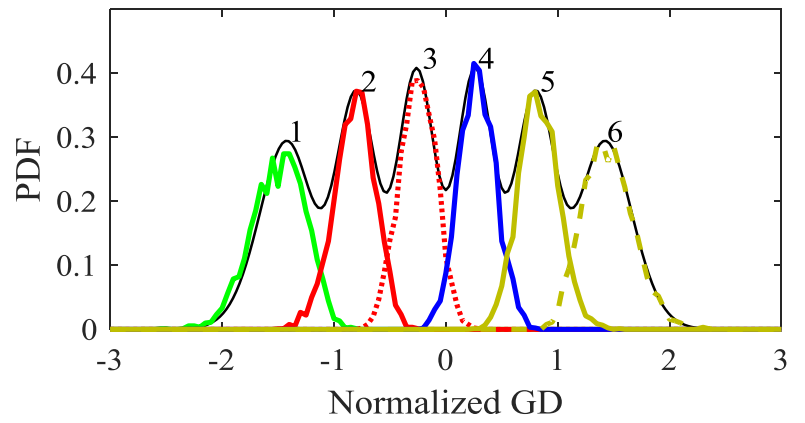

Fig. 9. Probability density function of the ordered normalized GDs $\left(\tau_{m} / \sigma_{g d}\right)$, obtained through simulation after $1000 \mathrm{~km}$, with different $X T_{\mathrm{LP}_{02}}$ values.

of the individual GDs evolution in Fig. 9, but similar matching between simulation and theory was obtained when considering dual-polarization. Fig. 9 (a) shows that for $-30 \mathrm{~dB} / 100 \mathrm{~m}$ the GDs of the PMs vaguely resemble the GDs of the LP modes given the impulse-like PDF of $\tau_{2}$ ("LP ${ }_{11 a}$ ") and $\tau_{3}$ (" $L P_{11 b}$ "). Further results for lower coupling values shown that all GDs present impulse-like PDFs. In Fig. 9 (b), for $-20 \mathrm{~dB} / 100 \mathrm{~m}$, the match between the simulated PDFs and the analytical PDF for strong coupling is good, even though the GDs have been normalized by different factors (24). Further increase of the coupling strength leads to improved matching between the simulated PDFs and the analytical $\mathrm{PDF}$, as observed in additional results.

In a MDM system for which the bandwidth of the PMs is much larger than the signal bandwidth, the digital equalizer must span a temporal memory at least as long as the the difference between the maximum and the minimum group delay $\left(\tau_{M}-\tau_{1}\right)_{\text {total. }}$. As shown in [8], the probability of having a GD spread lower than $x, P\left(\tau_{M}-\tau_{1} \leq x\right)$ - the cumulative distribution function, can be computed as a function of the joint probability of having all eigenvalues falling within an arbitrary interval $[x, y], P\left(\tau_{M} \leq x, \tau_{1} \geq y\right)$, this is:

$$
\begin{gathered}
P\left(\tau_{M}-\tau_{1} \leq x\right)=-\int_{-\infty}^{+\infty} \partial_{y} P\left(\tau_{M} \leq y+x, \tau_{1} \geq y\right) d y \\
P\left(\tau_{M} \leq x, \tau_{1} \geq y\right)=\int_{y}^{x} \ldots \int_{y}^{x} p_{M}(\boldsymbol{\tau}) d \tau_{1} \ldots d \tau_{M}
\end{gathered}
$$

According to [8], (27)-(29) can be evaluated using at least three methods: Fredholm determinant, Andréief identity or one approximation based on Tracy-Widom distribution. Finally, from (27), we can obtain the equalizer memory

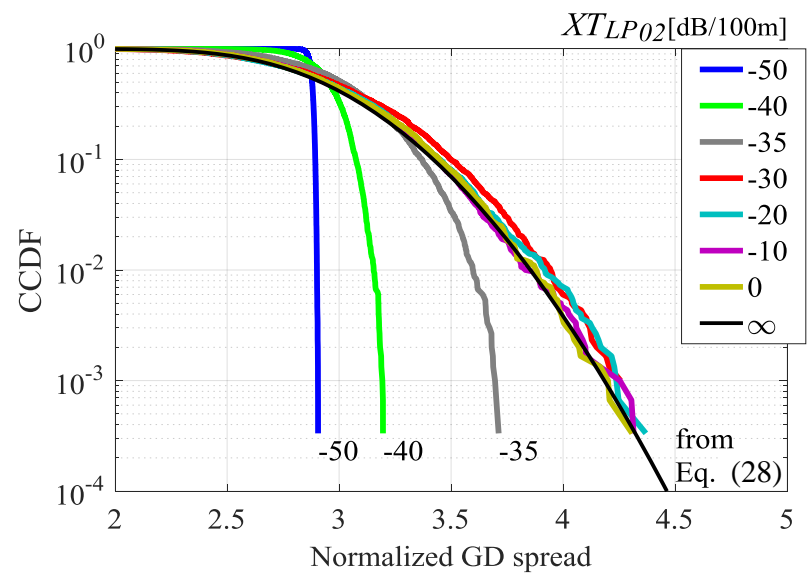

Fig. 10. Complementary cumulative distribution of the normalized GD spread, obtained through simulation after $1000 \mathrm{~km}$, with different $X T_{\mathrm{LP}_{02}}$.

length $x$ required to accommodate the GD spread with a given outage probability $p, \quad$ this is: $P\left(\tau_{M}-\tau_{1}>x\right)=p=1-P\left(\tau_{M}-\tau_{1} \leq x\right)$.

Fig. 10 shows the complementary cumulative distribution function (CCDF) of the normalized GD spread, $P\left[\left(\tau_{6}-\tau_{1}\right) / \sigma_{g d}>p\right]$, obtained through simulation after $1000 \mathrm{~km}$ for different coupling values (averaging over 6000 different realizations). These results were obtained for singlepolarization to be consistent with the PDFs in Fig. 9. Fig. 10 shows that for $X T_{\mathrm{LP}_{02}} \geq-30 \mathrm{~dB} / 100 \mathrm{~m}$ the CCDFs are very similar to the analytical approximation obtained for strong coupling (28) (dashed line). Conversely, for $X T_{\mathrm{LP}_{02}}$ lower than $-30 \mathrm{~dB} / 100 \mathrm{~m}$ the normalized GD spread is significantly smaller than the normalized GD spread for strong coupling. Finally, we can conclude that the required temporal equalizer memory length (in time units) to span a channel with an outage probability smaller than $10^{-4}$ is equal to $4.5 \sigma_{g d}$, for any coupling strength, where $\sigma_{g d}$ depends on the mode coupling strength, see (24).

\section{GD Statistics IN DElay-Managed Links}

In differential mode delay (DMD) managed spans, GD spread is reduced by cascading fibers with opposite sign DMD. In the absence of mode coupling, the GD spread at the end of the span would be zero. However, in the presence of coupling, the DMD compensation is no longer fully effective. In order to minimize the impact of coupling, the length of the segments over which DMD sign is inverted has to be made much smaller than the correlation length set by the coupling, $L_{c}$.

To compensate for linear mode coupling and group delay spread, MIMO-DSP can be used, but DSP complexity increases with the number of modes and the total GD spread. In order to minimise complexity, the total GD spread should typically be reduced to less than $10 \mathrm{~ns}$ [33].

For DMD-managed spans, where uncoupled GDs (per unit length) $\partial_{\omega} \boldsymbol{\beta}$ are a piecewise constant function of $z$ a general analytical solution of (23) for $E\left\{\|\boldsymbol{\tau}(z)\|^{2}\right\}$, rapidly becomes too complex as the number of fiber segments increases. Therefore, numerical integration should be performed as in [32].

In order to verify the deterministic numerical integration of 


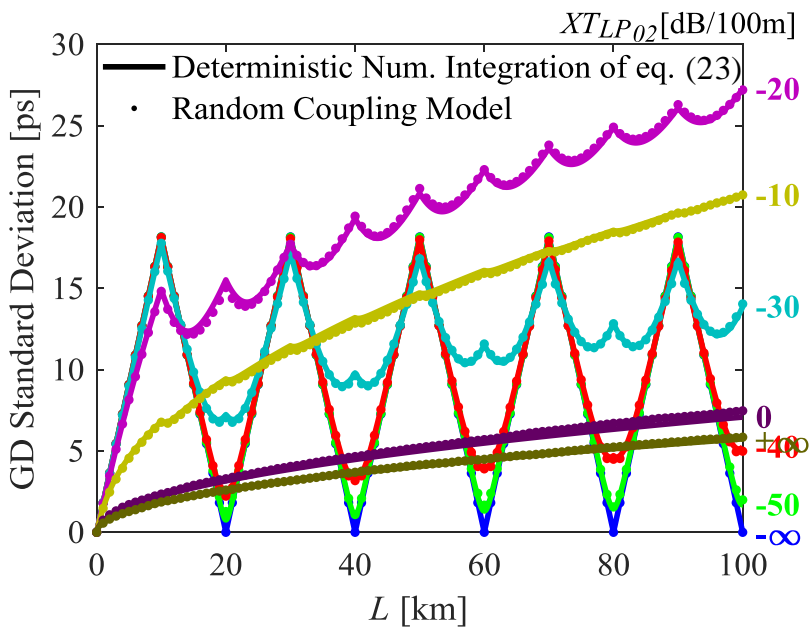

Fig. 11. Standard deviation of the GDs as a function of the propagation distance, for fibers with a compensation length of $20 \mathrm{~km}$ and different values of coupling strength.

(23) we made use of the multi-section model presented in section $\mathrm{V}$. The simulations considered that each span of length $L$ comprised $S$ segments, where each segment was itself composed by two fibres of length $L / S / 2$ with the same characteristics but opposite sign GD. The first fibre is the same presented in section V. The second fibre is not obtained through optimization but just by negating the GD vector, keeping the remaining characteristics of the first fibre.

Fig. 11 shows the evolution of the standard deviation of the GD vector $\left(\left[\tau_{1}, \tau_{2}, \ldots \tau_{12}\right]\right)$ with propagation distance, assuming compensation length of $20 \mathrm{~km}(10 \mathrm{~km}$ with the positive GD vector followed by $10 \mathrm{~km}$ with the negative GD vector), for different values of coupling strength. In Fig. 11, there are two sets of results, one obtained for transmission using the proposed multi-section model (dot markers) and one given by the deterministic numerical integration of (23) (full lines). A section length of $10 \mathrm{~m}$ was used as smaller section lengths generated similar results. In Fig. 11, we can observe a very good match between the deterministic numerical integration and the proposed multi-section model. It can be inferred from these results that the semi-analytical solutions in section IV multi-section model in section $\mathrm{V}$ are accurate under any coupling regime for DMD managed links.

In order to study a broader range of DMD scenarios, the GD vector was normalized by the highest GD value in the vector. Fig. 12 shows the combinations of $(D M D, X T)$ that allow for a GD spread lower than $800 \mathrm{ps}$ after $100 \mathrm{~km}$ with a probability higher than $95 \%$. In Fig. 12, for a given span configuration, GD spread is lower than 800 ps for $(D M D, X T)$ pairs below the respective curve. For non-DMD-managed spans, the maximum tolerable DMD increases with the coupling strength, being very low for weak coupling. For DMD-managed spans, as the number of segments increases, increasingly high DMD values are tolerable for weak coupling. For higher levels of coupling (above $-20 \mathrm{~dB} / 100 \mathrm{~m}$ ), the tolerable DMD converges to that of the non-DMDmanaged spans. Importantly, the tolerable DMD for the

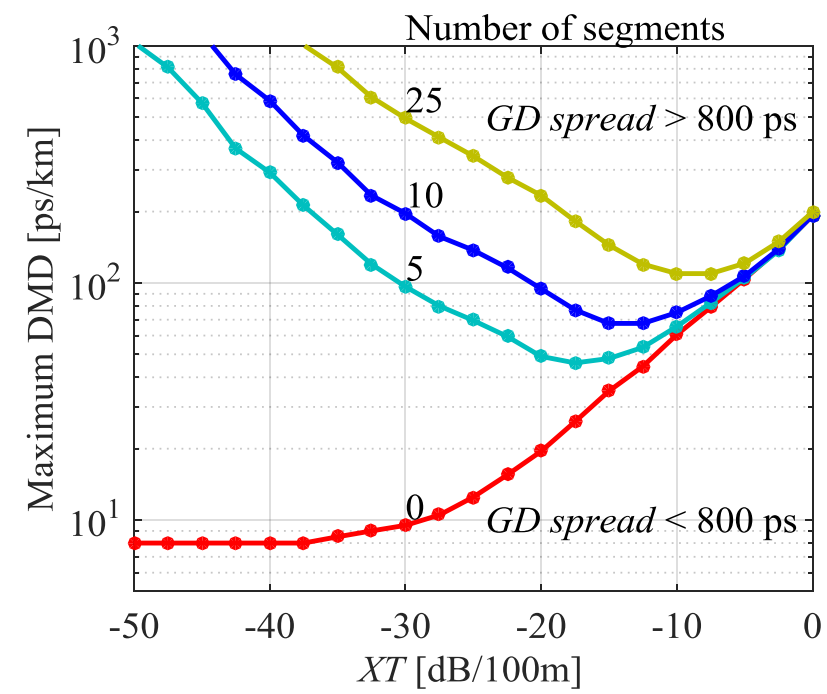

Fig. 12. Contour plot of the pairs $(D M D, X T)$ that allow for a GD spread lower than $800 \mathrm{ps}$ after $100 \mathrm{~km}$ with a probability higher than $95 \%$.

DMD-managed spans is always greater than or equal to the non-DMD-managed spans.

\section{CONCLUSION}

This paper proposes a semi-analytical solution method for the coupled linear differential equations that describe the linear modal coupling in FMFs. The analytical solutions obtained enable a time efficient computation of the mode coupling occurring after any fiber length in such a way that they can be integrated into a multi-section propagation model. This semi-analytical model is therefore a valuable alternative to the numerical methods which would not be practical due to computation time constraints. More importantly, the proposed model proved accurate against analytical predictions for the statistics of group-delays in few-mode fiber links, namely: standard deviation, probability density function, and cumulative distribution function. The proposed model proved accurate for different transmission lengths $10 \mathrm{~m}$-to$10,000 \mathrm{~km}$, in any coupling regime $-50 \mathrm{~dB} / 100 \mathrm{~m}$ to $0 \mathrm{~dB} / 100 \mathrm{~m}$, without and with GD management. Finally, the proposed semi-analytical solution method is an accurate and efficient tool for the modelling and development of future high-capacity multimode fiber systems.

\section{APPENDIX}

TABLE I

MATLAB CODE FOR FINDING EQUATIONS FOR $a_{m, r}$ WITH $M=3$

\begin{tabular}{|c|}
\hline $\begin{array}{l}A 1=\operatorname{sym}(' A 1(w) ') ; A 2=\operatorname{sym}\left(' A 2(w)^{\prime}\right) ; \\
A 3=\operatorname{sym}(' A 3(w) ') ;\end{array}$ \\
\hline 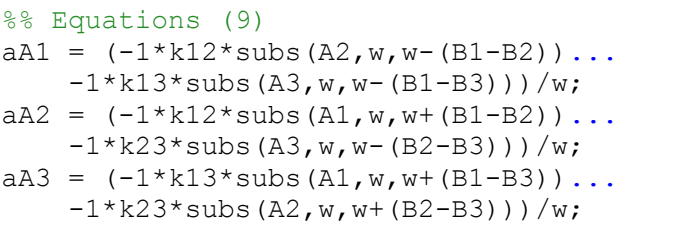 \\
\hline
\end{tabular}


응 Elimination of aA1 from aA2 and aA3

$\mathrm{xA} 2=\ldots$

( $\operatorname{subs}\left(\mathrm{aA} 2,{ }^{\prime} \mathrm{A} 1(\mathrm{w}+(\mathrm{B} 1-\mathrm{B} 2))\right.$ ', $\left.\left.\operatorname{subs}(\mathrm{aA} 1, \mathrm{w}, \mathrm{w}+(\mathrm{B} 1-\mathrm{B} 2))\right)\right)$; $\mathrm{xA} 3=\ldots$

$\left(\operatorname{subs}\left(\mathrm{aA} 3,{ }^{\prime} \mathrm{A} 1(\mathrm{w}+(\mathrm{B} 1-\mathrm{B} 3))\right.\right.$ ', $\left.\left.\operatorname{subs}(\mathrm{aA} 1, \mathrm{w}, \mathrm{w}+(\mathrm{B} 1-\mathrm{B} 3))\right)\right)$;

\% Find coefficient of $\mathrm{A} 2, \mathrm{~A} 3$ on the RHS of $x A 2, x A 3$

C xA2 $=\operatorname{subs}((x A 2-\operatorname{subs}(x A 2, ' A 2(w) ', 0)), ' A 2(w) ', 1)$;

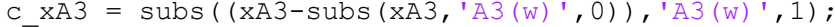

응 Passing the coefficients to the LHS

$\mathrm{xA} 2=\operatorname{subs}\left(\mathrm{xA} 2,{ }^{\prime A} 2(\mathrm{~W}) ', 0\right) /(1-\mathrm{C} \times \mathrm{xA})$;

$\mathrm{xA} 3=\operatorname{subs}\left(\mathrm{xA} 3,{ }^{\prime A} 3(\mathrm{w})^{\prime}, 0\right) /\left(1-\mathrm{C}_{-} \mathrm{xA} 3\right)$;

응 Elimination of A2 from $x A 3$

$\mathrm{zA} 3=\ldots$

subs $(x A 3, ' A 2(w+(B 2-B 3))$ ', $\operatorname{subs}(x A 2, w, w+(B 2-B 3)))$;

\% Equations (10) after passing A3 to the LHS of zA3 eq3 $=\left(1-\operatorname{subs}\left(z A 3, ' A 3(w)^{\prime}, 1\right)\right) ;$

응 Characteristic Polynomial Coefficients

[N3, D3] = numden (eq3);

$[\mathrm{C} 3, \mathrm{~T} 3]=\operatorname{coeffs}(\mathrm{N} 3, \mathrm{w})$;

$\%$ Final rearrangements

$\mathrm{C} 3=\operatorname{subs}(\mathrm{C} 3, \mathrm{~B} 1, \mathrm{~dB} 12+\mathrm{B} 2)$;

$\mathrm{C} 3=\operatorname{subs}(\mathrm{C} 3, \mathrm{~B} 2,-\mathrm{dB} 12+\mathrm{B} 1) ;$

$\mathrm{C} 3=\operatorname{subs}(\mathrm{C} 3, \mathrm{~B} 1, \mathrm{~dB} 13+\mathrm{B} 3)$;

$\mathrm{C} 3=\operatorname{subs}(\mathrm{C} 3, \mathrm{~B} 3,-\mathrm{dB} 13+\mathrm{B} 1)$;

$\mathrm{C} 3=\operatorname{subs}(\mathrm{C} 3, \mathrm{~B} 2, \mathrm{~dB} 23+\mathrm{B} 3)$;

$\mathrm{C} 3=\operatorname{subs}(\mathrm{C} 3, \mathrm{~B} 3,-\mathrm{dB} 23+\mathrm{B} 2)$;

$\mathrm{C} 3=$ simplify $(\mathrm{C} 3)$

similar for $\mathrm{C} 1$ and $\mathrm{C} 2 \%$

MATLAB CODE FOR FINDING EQUATIONS FOR $\left(d_{z}{ }^{r} A_{m}\right)_{z=0}$ WITH $M=3$

응 Boundary conditions

syms z

$\mathrm{A} 1=\operatorname{sym}\left({ }^{\prime} \mathrm{A} 1(\mathrm{z})^{\prime}\right) ; \mathrm{A} 2=\operatorname{sym}\left({ }^{\prime} \mathrm{A} 2(\mathrm{z})^{\prime}\right)$;

$\mathrm{A} 3=\operatorname{sym}\left({ }^{\prime} \mathrm{A} 3(\mathrm{z})^{\prime}\right)$ );

응 Equations (6)

$\mathrm{dA} 1=\ldots$

$-1 i *(\mathrm{k} 12 * \mathrm{~A} 2 * \exp (1 i * \mathrm{~dB} 12 * z)+\mathrm{k} 13 * \mathrm{~A} 3 * \exp (1 i *(\mathrm{~dB} 13) * z))$;

$\mathrm{dA} 2=\ldots$

$-1 i *(k 12 * A 1 * \exp (-1 i * d B 12 * z+k 23 * A 3 * \exp (1 i * d B 23 * z))$;

$\mathrm{dA} 3=\ldots$

$-1 i *(\mathrm{k} 13 * \mathrm{~A} 1 * \exp (-1 i * \mathrm{~dB} 13 * z+k 23 * \mathrm{~A} 2 * \exp (-1 i * \mathrm{~dB} 23 * z))$;

응 2nd-order derivative

$\mathrm{d} 2 \mathrm{~A} 1=\operatorname{diff}(\mathrm{dA} 1, \mathrm{z}) ;$

$\mathrm{d} 2 \mathrm{~A} 2=\operatorname{diff}(\mathrm{dA} 2, \mathrm{z})$

$\mathrm{d} 2 \mathrm{~A} 3=\operatorname{diff}(\mathrm{dA} 3, \mathrm{z}) ;$

\% Replacing known 1st-order derivatives

$\mathrm{d} 2 \mathrm{~A} 1=(\operatorname{subs}(\mathrm{d} 2 \mathrm{~A} 1, \operatorname{diff}(\mathrm{A} 1, \mathrm{z}), \mathrm{dA} 1)) ;$

$\mathrm{d} 2 \mathrm{~A} 1=(\operatorname{subs}(\mathrm{d} 2 \mathrm{~A} 1, \operatorname{diff}(\mathrm{A} 2, \mathrm{z}), \mathrm{dA} 2)) ;$

$\mathrm{d} 2 \mathrm{~A} 1=(\operatorname{subs}(\mathrm{d} 2 \mathrm{~A} 1, \operatorname{diff}(\mathrm{A} 3, \mathrm{z}), \mathrm{dA} 3))$

o similar for d2A2 and d2A2 \%

\% $\%$ rd-order derivative

$\mathrm{d} 3 \mathrm{~A} 1=\operatorname{diff}(\mathrm{d} 2 \mathrm{~A} 1, \mathrm{z})$;

$\mathrm{d} 3 \mathrm{~A} 2=\operatorname{diff}(\mathrm{d} 2 \mathrm{~A} 2, \mathrm{z}) ;$

$\mathrm{d} 3 \mathrm{~A} 3=\operatorname{diff}(\mathrm{d} 2 \mathrm{~A} 3, \mathrm{z})$;

응 Replacing known 1st-order derivatives

$\mathrm{d} 3 \mathrm{~A} 1=(\operatorname{subs}(\mathrm{d} 3 \mathrm{~A} 1, \operatorname{diff}(\mathrm{A} 1, \mathrm{z}), \mathrm{dA} 1)) ;$

$\mathrm{d} 3 \mathrm{~A} 1=(\operatorname{subs}(\mathrm{d} 3 \mathrm{~A} 1, \operatorname{diff}(\mathrm{A} 2, \mathrm{z}), \mathrm{dA} 2)) ;$

$\mathrm{d} 3 \mathrm{~A} 1=(\operatorname{subs}(\mathrm{d} 3 \mathrm{~A} 1, \operatorname{diff}(\mathrm{A} 3, \mathrm{z}), \mathrm{dA} 3)) ;$

\% similar for d $3 \mathrm{~A} 2$ and $\mathrm{d} 3 \mathrm{~A} 2$ \%

\%о Derivatives to be evaluated at $\mathrm{z}=0$

$\mathrm{BB} 1=\left[\operatorname{subs}\left(\mathrm{A} 1, \mathrm{z}^{\prime}, 0\right)\right.$

subs (dA1, ' $\left.z^{\prime}, 0\right)$

subs (d2A1, ' $\left.z^{\prime}, 0\right)$

subs (d3A1, ' $\left.\left.z^{\prime}, 0\right)\right]$;

o similar for BB2 and BB3

\section{RESEARCH DATA}

The Matlab scripts, source C-code, mex compiled C-code, and figure data points are available at http://doi.org/10.17036/researchdata.aston.ac.uk.00000206.

\section{REFERENCES}

[1] A. D. Ellis, J. Zhao and D. Cotter, "Approaching the Non-Linear Shannon Limit," Journal of Lightwave Technology, vol. 28, no. 4, pp. 423-433, 2010.

[2] R. J. Essiambre, G. Kramer, P. J. Winzer, et al., "Capacity Limits of Optical Fiber Networks," Journal of Lightwave Technology, vol. 28, no. 4, pp. 662-701, 2010.

[3] B. Inan, B. Spinnler, F. Ferreira, et al., "DSP complexity of modedivision multiplexed receivers," Opt. Express, vol. 20, pp. 1085910869, 2012

[4] A. Lobato, F. Ferreira, B.Inan, et al., "The Impact of differential mode delay on mode-division multiplexed coherent optical OFDM transmission," in Proc. OFC 2012, p. OTu2C.2.

[5] D. Marcuse, Theory of Dielectric Optical Waveguides, New York: Academic, 1974, Chap. 3 and 5

[6] K.-P. Ho and J. M. Kahn, "Statistics of Group Delays in Multimode Fiber with Strong Mode Coupling", Journal of Lightwave Technology, vol. 29, no. 21, pp. 3119-3128, 2011.

[7] K.-P. Ho and J. M. Kahn, "Linear propagation effects in mode-division multiplexing systems," J. Lightw. Technol., vol. 32, no. 4, pp. 614-628, Feb. 2014.

[8] K.-P. Ho and J. M. Kahn, "Delay-Spread Distribution for Multimode Fiber with Strong Mode Coupling", IEEE Photon. Technol. Lett., vol. 24, no. 21, pp. 1906-1909, 2012.

[9] C. Antonelli, A. Mecozzi, M. Shtaif, et al., "Stokes-space analysis of modal dispersion in fibers with multiple mode transmission," Opt. Express, vol. 20, pp. 11718-11733, 2012.

[10] A. Mecozzi, C. Antonelli, and M. Shtaif, "Intensity impulse response of SDM links," Opt. Express vol. 23, pp. 5738-5743, 2015.

[11] C. Antonelli, et al. "Random coupling between groups of degenerate fiber modes in mode multiplexed transmission, Opt. Exp., vol. 21, no. 8, pp. 9484-9489, Apr. 2013.

[12] C. Antonelli, et al "The delay spread in fibers for SDM transmission: Dependence on fiber parameters and perturbations," Opt. Exp., vol. 23, no. 3, pp. 2196-2202, Feb. 2015.

[13] Feihong Ye, S. Warm and K. Petermann, "Differential mode delay management in spliced multimode fiber transmission systems," in Proc. OFC 2013, p. OM3B.3.

[14] A. Juarez, E. Krune, S. Warm, et al., "Modeling of Mode Coupling in Multimode Fibers With Respect to Bandwidth and Loss," in Journal of Lightwave Technology, vol. 32, no. 8, pp. 1549-1558, 2014.

[15] F. Ferreira, S. Jansen, P. Monteiro and H. Silva, "Nonlinear SemiAnalytical Model for Simulation of Few-Mode Fiber Transmission," in IEEE Photonics Technology Letters, vol. 24, no. 4, pp. 240-242, 2012.

[16] F. Ferreira, P. Monteiro and H. Silva, "Semi-analytical model for linear modal coupling in few-mode fiber transmission," in Proc. ICTON 2012, p. Th.A1.5.

[17] F. Ferreira, D. Fonseca, A. Lobato, et al., "Reach Improvement of Mode Division Multiplexed Systems Using Fiber Splices," in IEEE Photonics Technology Letters, vol. 25, no. 12, pp. 1091-1094, 2013.

[18] L. Grüner-Nielsen, et al., "Few Mode Transmission Fiber With Low DGD, Low Mode Coupling, and Low Loss," J. Lightw. Technol., Vol. 30, no. 23, p. 3693, 2012.

[19] T. Mori, et al., "Low DMD Four LP Mode Transmission Fiber for Wide-band WDM-MIMO System," Proc. OFC 2013, p. OTh3K.1.

[20] R. Ryf, R.-J. Essiambre, A. H. Gnauck, S. Randel, et al., "Spacedivision multiplexed transmission over 4200-km 3-core microstructured fiber," in Proc. OFC 2012, p. PDP5C.2.

[21] N. Fontaine, et al., "Experimental investigation of crosstalk accumulation in a ring-core fiber," in Proc. PSSTMS 2013, p. TuC4.2.

[22] G. Agrawal, Nonlinear Fiber Optics, $3^{\text {rd }}$ ed. Academic Press, 2001, Chap. 2.

[23] L. Palmieri and A. Galtarossa, "Coupling effects among degenerate modes in multimode optical fibers," IEEE Photonics 6, 0600408, Dec. 2014

[24] M. Shemirani, W. Mao, R. A Panicker and J. M. Kahn, "Principal Modes in Graded-Index Multimode Fiber in Presence of Spatial- and 
Polarization-Mode Coupling", J. of Lightwave Technol., vol. 27, no. 10, pp. 1248-1261, 2009.

[25] F. Ferreira, S. Sygletos, and A. D. Ellis, "Impact of Linear Mode Coupling on the Group Delay Spread in Few-Mode Fibers," in Proc. OFC 2015, p. Tu2D.1.

[26] F. Ferreira, N. Suibhne, S. Sygletos and A. D. Ellis, "Few-mode fibre group-delays with intermediate coupling," in Proc. ECOC 2015, p. Th.1.6.1

[27] F. Ferreira, D. Fonseca and H. Silva, "Design of Few-Mode Fibers With M-modes and Low Differential Mode Delay," Journal of Lightwave Technology, vol. 32, no. 3, pp. 353-360, 2014.

[28] William H., S. Teukolsky, W. Vetterling, B. Flannery, Numerical Recipes in C: The Art of Scientific Computing, 3rd ed., New York: Cambridge University.

[29] J. Mathews, and K. Fink, Numerical Methods Using Matlab, Prencite Hall, Third Edition, 1999, Chap. 9.

[30] J. Noda, K. Okamoto, and Y. Sasaki, "Polarization-maintaining fibers and their applications," IEEE/OSA Journal of Lightwave Technology, vol. 4, no. 8, pp. 1071-1089, 1986.

[31] S. Fan and J. Kahn, "Principal modes in multimode waveguides," Opt. Lett., vol. 30, no. 2, p. 135, 2005.

[32] S. Ö. Arik, K.-P. Ho and J. M. Kahn, "Delay Spread Reduction in Mode-Division Multiplexing: Mode Coupling versus Delay Compensation", J. of Lightwave Technol., vol. 33, no. 21, pp. 45044512, 2015.

[33] S. Randel, R. Ryf, A. Gnauck, et al., "Mode-multiplexed 6×20-GBd QPSK transmission over 1200-km DGD-compensated few-mode fiber," in Proc. OFC 2012, p. PDP5C.5. 